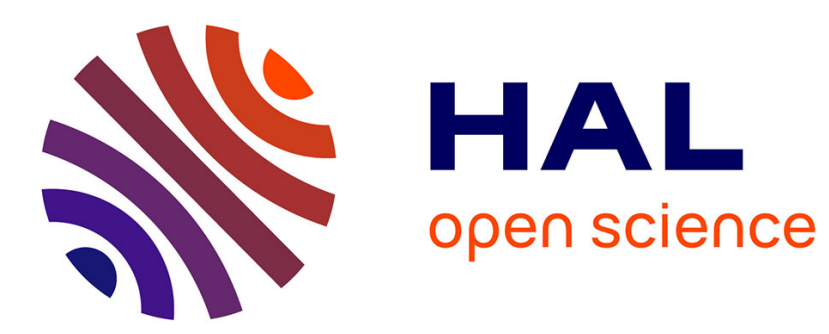

\title{
Sur les phénomènes d'induction
}

\author{
L. Mouton
}

\section{To cite this version:}

L. Mouton. Sur les phénomènes d'induction. J. Phys. Theor. Appl., 1877, 6 (1), pp.5-16. 10.1051/jphystap:0187700600500 . jpa-00237358

\section{HAL Id: jpa-00237358 https://hal.science/jpa-00237358}

Submitted on 1 Jan 1877

HAL is a multi-disciplinary open access archive for the deposit and dissemination of scientific research documents, whether they are published or not. The documents may come from teaching and research institutions in France or abroad, or from public or private research centers.
L'archive ouverte pluridisciplinaire HAL, est destinée au dépôt et à la diffusion de documents scientifiques de niveau recherche, publiés ou non, émanant des établissements d'enseignement et de recherche français ou étrangers, des laboratoires publics ou privés. 


\section{JOURNAL}

\section{E PHYSIQUE}

\section{THÉORIQUE ET APPLIQUÉE.}

\section{SUR LES PHÉNOMÉNES D'INDUGTION;}

PAR M. L. MOUTON.

Au moment de la fermeture et à celui de la rupture du courant inducteur, une bobine induite est le siége de mouvements électriques qui, depuis Faraday, ont été constatés et étudiés par l'un ou l'autre des procédés suivants : $\mathrm{I}^{\mathrm{o}}$ les extrémités du fil induit sont reliées à un galvanomètre; ce fil constitue ainsi un circuit fermé dans lequel le galvanomètre indique le passage de courants de durée très-courte dits courants d'induction; $2^{\circ}$ ces extrémités sont fixées à une spirale magnétisante; les phénomènes sont alors accusés par l'aimantation des aiguilles d'acier placées dans la spirale; $3^{\circ}$ ces mêmes extrémités sont mises en relation par le corps d'un animal dont les commotions sont le signe des mouvements électriques $; 4^{\circ}$ enfin les pôles de la bobine sont séparés par un espace d'air suffisamment faible, ou par une colonne de gaz raréfiés, tels que les fluides ébranlés puissent les franchir sous la forme complexe de l'étincelle ou de la lumière dite des tubes de Geissler.

Je me suis proposé de résoudre la question suivante: Les extrémités de la bobine induite étant complétement isolées, de façon qu'il ne puisse se produire ni courant en circuit, ni étincelles, de quels phénomènes le fil induit est-il le siége? En précisant davantage et me bornant au cas de la rupture du courant inducteur, 
pendant le passage de ce courant, les extrémités du fil induit ne présentent aucune différence de tension ( $\left.{ }^{1}\right)$; la même chose a lieu quelques instants après la rupture. Par quelles valeurs successives a passé cette différence de potentiel ainsi partie de zéro et revenue à zéro?

Je décrirai d'abord sommairement le dispositif expérimental employé, puis les résultats obtenus $\left({ }^{2}\right)$.

Disjoncteur. - Il se compose d'abord de trois roues en cuivre de I décimètre de diamètre, $\mathrm{A}, \mathrm{B}, \mathrm{B}$ ( $f g$. I ), montées sur le

Fig. I.

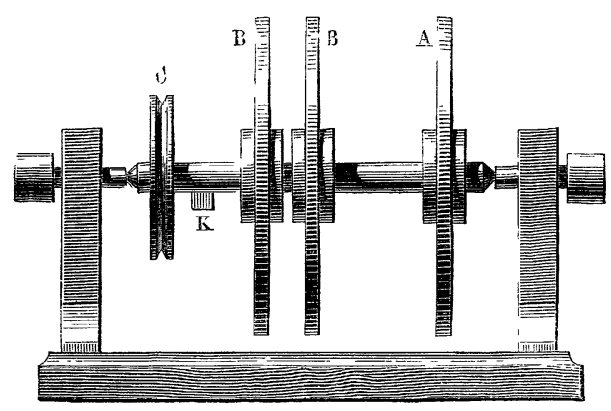

même arbre horizontal et soigneusement isolées de cet arbre par des pièces d'ébonite. La roue $\mathrm{A}$ produit la fermeture et la rupture du courant inducteur. A cet effet, elle présente un petit filet excentrique $a(f i g .2)$, qui vient à chaque tour frotter contre une pièce en bronze $b$ taillée en couteau. Cette pièce, constamment poussée par un ressort, est tenue à distance de la roue A par une vis buttante et ne communique avec elle qu'au passage du filet excentrique. La pièce $b$ fait partie d'un système très-solide $\mathrm{D}$, isolé du

( ${ }^{1}$ Il est bien entendu que la tension dont il sera toujours question dans cet article est celle qui se constate par communication métallique lointaine, qui est indépendante du point touché du conducteur, la tension de Volta, comme l'appelle bien justement M. Mascart (Traité d'électricité statique, 1876, Préface), ou enfin le potentiel pour ceux que n'effraient pas trop les considérations mathématiques a priori.

( ${ }^{2}$ ) Cet article, ainsi que les figures qui s'y rapportent, est extrait d'une thèse que je viens d'avoir l'honneur de soutenir devant la Faculté des Sciences de Paris. Je prie le lecteur d'y recourir pour tout ce qui lui paraitra ici trop succinctement exposé. (Ann. de l'École Normale, $2^{\text {e }}$ série, t. VI ; Paris, Gauthier-Villars.) 
reste de l'appareil et dans lequel elle peut s'élever ou s'abaisser verticalement de quantités soigneusement mesurées par une vis micrométrique $c$ graduée au $\frac{1}{s_{0}}$ de millimètre. Le courant inducteur entrant par la borne $d$ passe dans la roue $\mathrm{A}$ par le ressort frotteur $\alpha$

Fig. 2.

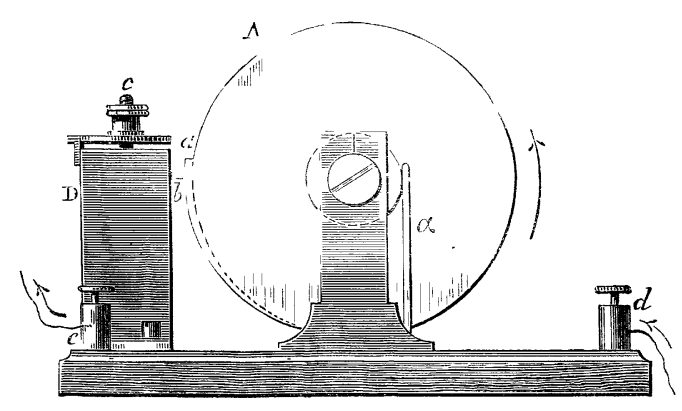

et, pendant le contact de la pièce $b$ avec le filet $a$, gagne le système D et retourne à la pile par la borne $e$.

Les roues B, B sont identiques. Chacune d'elles présente un couteau $f(f g .3)$ parallèle à l'axe de rotation, porté à l'extrémité

Fig. 3.

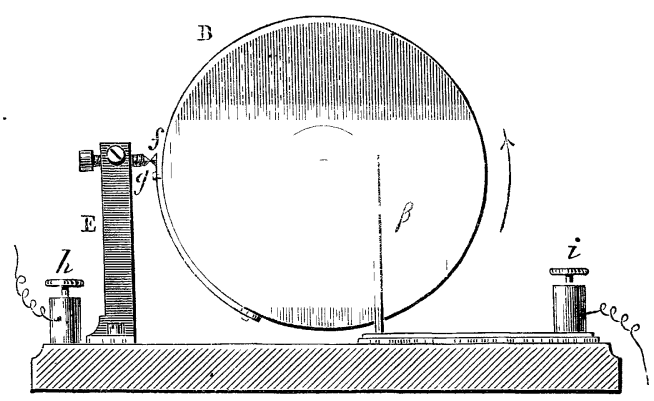

d'un long ressort couché parallèlement à la circonférence de la roue et qu'empêchent de vibrer des vis buttantes le condamnant à n'effectuer que des mouvements d'amplitude extrêmement faible. A chaque révolution, le couteau touche une pointe fixe $g$ portée sur une colonne isolée et mise en relation avec les quadrants d'un élec- 
tromètre par un fil partant de la borne $h$. Les roues $\mathrm{B}, \mathrm{B}$ communiquent en outre constamment, par deux frotteurs $\beta$ isolés du reste de l'appareil, avec les bornes $i$, mises par un fil en relation métallique avec les extrémités du fil induit. Au moyen de la loupe, contrôlée par un procédé électrique particulier, je m'assurais que les contacts des couteaux $f$ avec les pointes $g$ avaient bien lieu au même instant. Si l'on se reporte à la description du système $\mathrm{D}$, on voit alors que, en faisant monter ou descendre la pièce $b$ au moyen de la vis micrométrique $c$, on peut faire que l'instant du double contact des couteaux $f$ et des pointes $g$ se rapproche ou s'éloigne de quantités bien déterminées de celui de la rupture métallique du courant inducteur.

Ces quantités, mesurées par la vis micrométrique, se traduisent aisément en temps si l'on connaît la vitesse de rotation de l'appareil. Pour mesurer cette vitesse, une came K (.fg. I) pressant sur un ressort fermait, à chaque tour de l'appareil, un courant spécial communiquant avec un système inscripteur dont il sera dit un mot tout à l'heure.

Enfin la poulie C ( $f g$ g. I ) était reliée à l'arbre du moteur par une corde qui communiquait le mouvement à l'appareil.

Cet instrument a été construit par MM. Brünner frères.

Moteur et régulateur. - Je me suis servi pour moteur d'une petite machine Gramme dans le fil de laquelle était lancé le courant de deux ou trois éléments Bunsen. Des irrégularités dans le mouvement, dues probablement aux variations de la pile, m'ont conduit à y adjoindre le régulateur représenté $(f i g .4)$ et applicable à tout moteur électromagnétique. C'est le système à force centrifuge de Watt, prenant son mouvement par une corde sur l'arbre même de la machine. Au sommet A du losange articulé où se trouve fixé dans le régulateur des machines à feu l'anneau portant le bras du levier qui doit augmenter ou diminuer l'entrée de la vapeur dans le cylindre, est fixée une tige horizontale faisant corps avec cet anneau et guidée par deux baguettes verticales $a, a$, l'empêchant de faire autre chose que s'élever ou s'abaisser parallèlement à elle-même, selon que les boules s'élèvent ou s'abaissent. Aux deux extrémités $b, b$ de cette tige horizontale sont fixés deux cylindres B, B en caoutchouc durci, sur chacun desquels est enroulé 
en hélice un fil de fer de $\frac{1}{2}$ millimètre de diamètre environ. Ces cylindres plongent dans des vases remplis de mercure; le fil de fer enroulé se trouve ainsi communiquer par une extrémité avec le mercure, par l'autre avec l'ensemble métallique de l'appareil. Le courant qui fait mouvoir le moteur, au lieu de se rendre direc-

Fig. 4.

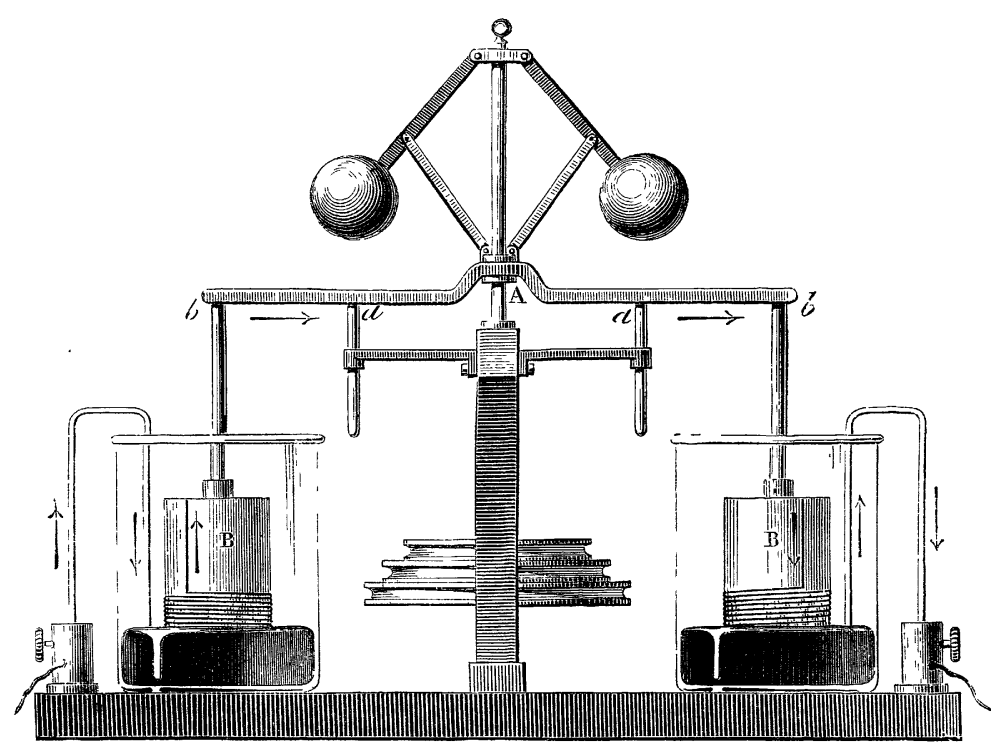

tement dans celui-ci, est d'abord lancé dans le mercure des vases, de là il passe dans les spires émergées du fil de fer, puis dans la machine.

On comprend qu'un accroissement de vitesse, par exemple, de la machine se traduit immédiatement par la sortie d'une ou plusieurs spires du mercure, d'où une augmentation de résistance (la résistance d'une spire était égale au $\frac{1}{10}$ environ de la résistance totale du circuit) et par suite un affaiblissement du courant moteur.

Cet appareil a été construit par M. Ducretet.

Inscripteur du mouvement. - Il était important de savoir d'abord jusqu'où allait la régularité du mouvement. Pour cela, j'adaptai au disjoncteur le petit appareil représenté $(f i g .5)$. La came K, dont 
on a vu la place dans le disjoncteur, venait à chaque tour de celuici presser le ressort $\mathrm{R}$ et amener le contact entre la pointe $n$ et l'enclume $m$. Le courant d'une pile spéciale, alors fermé, mettait en action un électro-aimant inscripteur ordinaire. Ce dernier traçait une courbe sur un cylindre tournant recouvert de papier noirci, concurremment avec celle des vibrations d'un diapason de

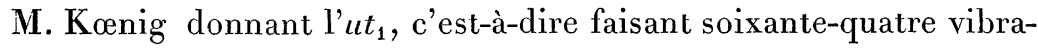

Fig. 5.

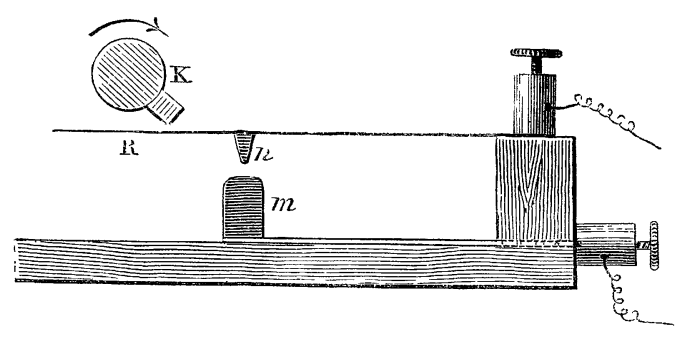

tions complètes par seconde. Je me suis borné à compter le nombre des vibrations du diapason correspondant à vingt tours du disjoncteur, et, pour citer les chiffres d'une série, je l'ai trouvé compris toujour's entre ıo et Ior; je puis donc affirmer que le nombre $n$ de tours par seconde était compris ce jour-là entre $\frac{2 v \times 64}{100}=12,8$ et $\frac{20 \times 64}{10 \mathrm{I}}=\mathrm{I}_{2}, 7$, et que par conséquent la vitesse ne variait pas, dans un intervalle de plus de quatre minutes, du centième de sa valeur moyenne.

Une fois assuré de la régularité du mouvement, je substituai au cylindre tournant un simple récepteur Morse, dont j’avais comparé la marche à un chronomètre de Winnerl. Il était facile de déduire du nombre de points compris dans une bande de longueur donnée la valeur absolue de la vitesse à chaque expérience.

Électromètre et sa graduation. - L'instrument de mesure était un électromètre à quadrants de M. Thomson, fabriqué chez MM. EIliott, de Londres; il présente le système de réflecteur communément employé dans les galvanomètres de $\mathbf{M}$. Thomson : un petit miroir concave de 80 centimètres de rayon fixé à l'aiguille. En regard et à 80 centimètres, se trouve une planchette portant une échelle 
horizontale, et au-dessouș de cette échelle une fente que les Anglais éclairent par une lampe à pétrole. L'image de la fente et même d'un fil qu'on peut tendre en son milieu se produit très-nette sur l'échelle. L'approximation est incontestablement moindre que celle que donnent les lunettes; mais ce procédé présente en revanche l'avantage de n'exiger qu'un coup d'œil que l'on peut jeter rapidement sur l'échelle, tout en opérant. A ce point de vue surtout, je me permets de le recommander aux chercheurs.

L'aiguille demeurait chargée par la communication du condensateur avec un des pôles d'une pile ouverte qui n'a jamais dépassé cinquante éléments zinc-platine-eau.

Cette méthode de charge est très-commode; mais elle demande à être entourée de quelques précautions.

D'abord il faut se prémunir contre la variabilité possible de cette pile; je l'ai fait en m'en référant tous les jours à une batterie de vingt éléments Daniell, soigneusement montés avec des dissolutions toujours identiques de sulfate de zinc et de sulfate de cuivre; je considère ainsi comme négligeables, d'après M. Thomson, les variations de cet élément type que je prends comme unité de force électromotrice ou de différence de potentiel $\left({ }^{1}\right)$.

Une cause d'erreurs bien plus considérables peut résulter de la faiblesse de cette charge, à laquelle peuvent devenir aisément comparables les différences de potentiel que l'on veut mesurer.

Dans ce cas une même différence de potentiel des secteurs peut être accusée par des déviations absolument différentes selon la valeur absolue de ces potentiels. Une formule donnée dans le Journal de Physique par M. Mascart $\left({ }^{2}\right)$ m'a permis d'étudier complétement l'instrument sous ce point de vue, et de tirer même avantage de ce qui paraissait tout d'abord une infériorité.

Si $\mathrm{V}$ est le potentiel fixe de l'aiguille, $\mathrm{V}_{1}$ et $\mathrm{V}_{2}$ ceux des secteurs, le moment du couple qui tend à dévier l'aiguille, supposée retenue au zéro a une valeur de la forme

$$
\mathbf{M}=l \mathbf{V}\left(\mathbf{V}_{1}-\mathbf{V}_{2}\right)+h\left(\mathbf{V}_{2}^{2}-\mathbf{V}_{1}^{2}\right),
$$

( $\left.{ }^{1}\right)$ Voir Thouson, Reprint of papers on electrostatics and magnesism; London, $187_{72}$, p. ${ }^{2} 6$.

(") Journal de Physique, t. IV, p. 324 et suiv. (Extrait d'un T'raité d'électricité statique aujourd'hui paru.) 
où $l$ et $h$ sonl deux constantes dépendant de l'instrument. J'ai d'abord vérifié la conséquence suivante de cette formule : si les points reliés aux secteurs sont les pôles d'une pile ouverte de force électromotrice totale $A$, si l'on appelle $M_{2}$ le moment du couple de déviation quand le pôle positif communique au sol, $\mathrm{M}_{3}$ ce moment quand c'est le pôle négatif, et $\mathbf{M}_{1}$ quand on met au sol le milieu de la pile, on a

$$
\begin{aligned}
& \mathbf{M}_{2}=l \mathbf{V A}+h \mathbf{A}^{2}, \\
& \mathbf{M}_{3}=l \mathbf{V A}-h \mathbf{A}^{2},
\end{aligned}
$$

et

$$
\mathbf{M}_{1}=l \mathbf{V A}=\frac{\mathbf{M}_{2}+\mathbf{M}_{3}}{2}
$$

c'est-à-dire que le moment dans le dernier cas est la moyenne arithmétique des deux autres.

Les mêmes piles ouvertes m'ont permis de vérifier la seconde conséquence que voici :

Soit $\mathbf{M}_{1}$ le moment correspondant au potentiel $\mathbf{V}$ de l'aiguille, et $\mathrm{V}_{1}, \mathrm{~V}_{2}$ des secteurs, on a

$$
\mathbf{M}_{1}=l \mathbf{V}\left(\mathrm{V}_{1}-\mathrm{V}_{2}\right)+h\left(\mathrm{~V}_{2}^{2}-\mathrm{V}_{1}^{2}\right) .
$$

Changeons $\mathrm{V}$ en $-\mathrm{V}$, en intervertissant les pôles de la pile de charge: le nouveau moment $M_{2}$ sera

$$
\mathrm{M}_{2}=-l \mathrm{~V}\left(\mathrm{~V}_{1}-\mathrm{V}_{2}\right)+h\left(\mathrm{~V}_{2}^{2}-\mathrm{V}_{1}^{2}\right),
$$

qui pourra être de signe contraire ou de même signe que $\mathbf{M}_{1}$. Retranchons $M_{2}$ de $M_{1}$, ce qui reviendra, au cas le plus fréquent où les déviations sont de sens contraire, à ajouter leurs valeurs absolues; on a

$$
\frac{M_{1}-M_{2}}{2}=l \mathrm{~V}\left(\mathrm{~V}_{1}-\mathrm{V}_{2}\right),
$$

c'est-à-dire la déviation qu'aurait fournie la même différence de potentiel avec deux potentiels égaux et de signe contraire.

Voici un tableau obtenu avec une pile de charge de cinquante éléments zinc-cuivre-eau, et une pile d'essai de trente éléments zinc-platine-eau. 
INDUCTION.

\begin{tabular}{|c|c|c|c|c|}
\hline $\begin{array}{l}\text { CHarge } \\
\text { de l'aiguille. }\end{array}$ & $\begin{array}{l}\text { DÉVIATION } \\
\text { droite. }\end{array}$ & $\begin{array}{l}\text { DÉVIATIOY } \\
\text { gauche. }\end{array}$ & SOMME. & MOYENNE. \\
\hline \multicolumn{5}{|c|}{ Milieu au sol. } \\
\hline - & I 80 & $19^{5}$ & $3-5$ & \multirow{2}{*}{366,5} \\
\hline+ & 170 & I 88 & 358 & \\
\hline \multicolumn{5}{|c|}{ Pôle - au sol. } \\
\hline - & 220 & 220 & 440 & \multirow{2}{*}{363,5} \\
\hline$T$ & 150 & 137 & 287 & \\
\hline \multicolumn{5}{|c|}{ Pôle + au sol. } \\
\hline+ & 215 & 225 & 440 & \multirow{2}{*}{365} \\
\hline - & 150 & 160 & $29^{\circ}$ & \\
\hline \multicolumn{5}{|c|}{$10^{\mathrm{e}}$ élément du côté négatif au sol. } \\
\hline+ & $x>0$ & 1,5 & 345 & \multirow{2}{*}{362,5} \\
\hline - & 185 & $19 \tilde{3}$ & 380 & \\
\hline
\end{tabular}

On voit que les nombres de la colonne des moyennes ne diffèrent pas les uns des autres de plus de $\frac{\dot{9} 0}{90}$ de leur valeur.

Ce fait ètabli, j’ai dressé une courbe de graduation de l'instru-

Fig. 6 .

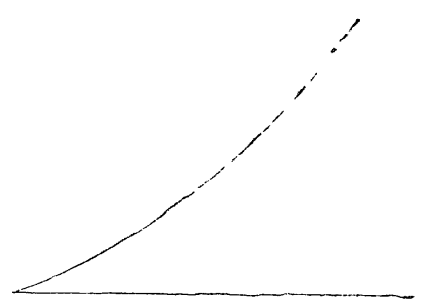

ment en reliant aux secteurs des séries successives de ı o, 20,3o,... éléments de pile, à milieu fixé au sol, calquant absolument le procédé de graduation du galvanomètre par la lentille à secteurs de MM. de la Provostaye et Desains; la $f i g .6$ est une réduction 
au $\frac{1}{20}$ de la courbe ainsi tracée. J'ai placé entre la pile de charge et l'aiguille un commutateur à bascule permettant une rapide interversion des pôles, et j'ai pu avoir ainsi, par une double mesure, à la fois des nombres toujours comparables entre eux, et être renseigné en même temps sur la valeur absolue des potentiels en jeu.

Soient A l'une de ces déviations, moyenne des deux observations, B l'ordonnée correspondante de la courbe de l'électromètre; les vingt Daniell donnaient ce jour une déviation $a$ à laquelle correspondait une ordonnée $b$; la différence du potentiel est $\frac{20}{b} \mathrm{~B}$, l'élément Daniell étant l'unité.

Appareil d'induction. - J'ai employé les deux bobines induites suivantes: la première de diamètre extérieur $7^{\mathrm{c}}, 5$ environ, longueur i 5 centimètres; le fil y fait $\mathrm{i} 386$ o tours avec un diamètre de $\frac{1}{4}$ de millimètre, et une longueur approximative de 2500 mètres. Sa résistance mesurée directement est $942 \mathrm{Ohms}$; elle provient d'un schlitten-apparat de du Bois-Reymond, fabriqué à Heidelberg. La seconde bobine m'a été gracieusement offerte par M. Ruhmkorff; elle a les mêmes dimensions, avec 7260 spires d'un fil de $\frac{2}{5}$ de millimètre de diamètre, environ I 200 mètres de long; sa résistance est $164 \mathrm{Ohms}$. Elle est formée de deux bobines qui peuvent s'accoupler, soit par le bout intérieur du fil enroulé, soit par le bout extérieur.

J'ai construit quatre bobines inductrices qui pénétraient exactement à l'intérieur de l'une ou de l'autre des bobines induites dont elles avaient la longueur: elles sont recouvertes d'un fil de cuivre de I millimètre de diamètre; l'une en a une seule épaisseur, les autres deux, trois et quatre; elles ne présentent aucune pièce de fer à l'intérieur.

Courant inducteur. - Il était fourni par un élément Daniell moyen modèle, plus ou moins complétement rempli avec des dissolutions bien pures de sulfate de zinc et de sulfate de cuivre; la résistance en était mesurée par un procédé indiqué dans le Journal de Physique $\left({ }^{1}\right)$; un rhéocorde de Poggendorff servait à modifier

(1) Journal de Physique, t. V, p. 144. 
l'intensité du courant, dont une fraction convenable était lancée à volonté dans un galvanomètre de Weber.

Discussion de la méthode; résultats. - Au point de vue théorique, la méthode employée ne semble pas comporter une longue discussion; voici sur quoi elle s'appuie : $\mathbf{I}^{0}$ après chaque rupture, l'état électrique du fil induit passe identiquement par les mêmes phases : les commutateurs, rhéotropes, disjoncteurs, etc., employés depuis Masson, n'auraient sans cela aucun sens ; $2^{\circ} \mathrm{la}$ différence de potentiel que présentent les deux extrémités du fil induit à un temps $t$ déterminé après la rupture du courant inducteur, elles la reprendront par conséquent à tous les temps correspondants. $\mathrm{Si}$, à chacun de ces temps $t$, elles sont mises en relation métallique avec deux corps A eı B et que la durée de cette relation soit assez courte pour que la différence de potentiel propre au temps $t$ n'ait pas changé d'une façon sensible, au bout d'un certain nombre de contacts, qui dépendra de la capacité relative du fil induit et des corps $\mathrm{A}$ et $\mathrm{B}$, ces derniers auront pris et conserveront la différence de potentiel des extrémités du fil correspondant au temps $t$ considéré, et à partir de ce moment ces contacts ne troubleront plus le phénomène de l'induction que dans les mesures des pertes que pourront éprouver les conducteurs A et B. Ces pertes peuvent être considérées comme nulles avec des contacts se succédant à moins de $\frac{1}{10}$ de seconde d'intervalle, et quand il s'agit de potentiels ne dépassant pas un ou deux cents Daniell.

Si l'on se reporte aux analogies établies et si souvent invoquées par M. Thomson entre la propagation de la chaleur et celle de l'électricité, le potentiel électrique et la température, ou plus simplement à la considération de la charge à refus des anciens électriciens, ce qui précède paraît incontestable.

Au point de vue pratique, il ne me reste que peu de chose à ajouter à la description des appareils. Voici comment se faisait chaque série d'expériences: d'abord toutes les pointes et couteaux servant de contacts étaient soigneusement examinés à la loupe et retouchés quand ils atteignaient, par l'usure, une épaisseur de $\frac{1}{10}$ de millimètre au plus. Je m'assurais ensuite que les deux couteaux $f$ ( $f g .3$ ) et les pointes $g$ se touchaient bien au même instant; pour cela les extrémités d'une pile ouverte étaient d'abord mises direc- 
tement en relation avec les quadrants de l'électromètre et l'on notait la déviation ; je les reliais alors aux bornes et j'amenais les couteaux au contact des pointes; l'un des couteaux était retouché jusqu'à ce que la déviation fût la même. Le système d'induction étant ensuite mis en place, je plaçais le couteau $b$ de façon que le double contact précédent eût lieu lorsque le courant inducteur était encore fermé : dans toutes mes expériences l'électromètre resta alors au zéro, ce qui indique que le temps pendant lequel se trouvait fermé le courant inducteur était plus que suffisant pour que tous les phénomènes d'induction correspondant à son établissement fussent éteints. Je faisais marcher enfin degré par degré la vis micrométrique $c$ et je notais les données correspondantes de l'électromètre. On se rappelle qu'un télégraphe Morse me donnait le nombre $n$ de tours du disjoncteur par seconde. Il est aisé d'en conclure le temps correspondant au déplacement d'une division de la vis micrométrique. Le diamètre des roues étant de I décimètre, la circonférence est $3 \times 4$ millimètres; à $n$ tours par seconde, il défilait donc devant le couteau 3 I $4 n$ millimètres par seconde; d'où le temps correspondant à $\frac{1}{50}$ de millimètre était $\frac{\mathrm{I}}{314 \times 50 \times n}$. Le nombre $n$ a été en moyenne de ı, ce qui donne pour le temps considéré $\frac{\mathrm{I}}{157000}$ de seconde. Avec la même vitesse, on voit que la durée des contacts des extrémités du fil avec l'électromètre était inférieure à $\frac{1}{314 \times 10 \times 10}$ ou $\frac{\mathrm{r}}{31400}$ de seconde. 